\title{
The Labours of Zeno - a Supertask indeed?
}

\section{Introduction:}

The last 60 years have produced a prolific literature constituting the so-called supertask debate - the debate whether an infinite sequence ${ }^{1}$ of actions or operations carried out in a finite interval of time is indeed possible. ${ }^{2}$ With the exception of J.F. Thomson and Max Black, people involved in this discussion usually claim that the original founder of the debate was Zeno with his dichotomy paradox. ${ }^{3}$ This paradox seems to pose the problem that the finite process of continuously running a finite distance requires covering infinitely many spatial parts, and thus performing infinitely many tasks, a supertask.

In this paper, I show that (a) Zeno's paradox in fact does not present us with a supertaks but rather raises a very different worry. I will claim that Thomson and Black are right in denying Zeno to be the originator of the supertask debate, but for reasons different to the ones they provide us with. In a second step, I demonstrate that (b) comparing Zeno's paradox with a paradigmatic supertask can nevertheless be instructive, since it forces us to make explicit some pre-conditions on which the very notion of a supertask rests and to examine whether these conditions are indeed given in the case of a continuous run.

Understanding a continuous run as a series of tasks, and ultimately a supertask, is prepared by the so-called at-at-theory of motion - the theory that claims that motion consists in nothing but being at a particular point at a particular time. But a closer look at Zeno's paradox suggests that, in contrast to common scholarly opinion, we should not understand continuous motions as a series of tasks. For the requirements for supertasks and for continuous finite runs (whether or not presented by Zeno) are genuinely different and not reducible to each other; we will see that they have a different mereological structure. And indeed these differences suggest that we may want to re-think our usage of the at-at-theory for our account of motion.

\section{Supertasks}

The debate whether supertasks are possible is not a debate about Hercules and his

\footnotetext{
${ }^{1}$ This account has been determined further as "a numerable infinite sequence" in recent times, in order to distinguish supertasks from hypertasks, which are non-numerable infinite sequences.

${ }^{2}$ Originally, the debate started in the journal Analysis, while many of the later articles in this debate were published in the British Journal for the Philosophy of Science.

${ }^{3} \mathrm{He}$ is either seen as simply inventing supertasks or as showing that supertasks are impossible.
} 
labours. The question of the possibility of supertasks revolves not around tasks we might think divinities or superheroes to be engaged with. Rather, the core idea of a supertask is an infinite series of tasks to be performed either by human beings or by machines set up by human beings, or, more recently, by physical particles. The series of tasks is set up in such a way that the time between two tasks gets successively lesser - and in recent versions, usually each task gets successively smaller - so that the time in between approaches zero. Thus, this infinity of tasks should be performed in a finite time, and no superpower should be needed.

To illustrate such a supertask, let us look briefly at a very simple so-called "infinity machine", i.e. a machine which is supposed to perform such a supertask: the reading lamp Thomson introduced as an infinity machine. ${ }^{4}$ This lamp has one switch to turn the light on and off. If we look at it over the period of one minute, we may imagine experiencing the following scenario: the button of the lamp is pressed to switch on the light after $1 / 2$ a minute, it is pressed again to turn off the light after another $1 / 4$ of a minute, pressed again to turn on the light after $1 / 8$ of a minute, etc. Thus, after one minute, the task has been performed infinitely many times.

Are such supertasks logically possible but physically impossible? Or possible or impossible in both respects? The supertask debate has seen defenders of all three positions. ${ }^{5}$

The problem of the possibility of supertasks has often been situated within the discussion about the degree of determinism in a Newtonian framework: ${ }^{6}$ is a Newtonian framework thoroughly deterministic in such a way that every state of affairs can be deduced from the preceding ones, or do we also have to allow for some indeterminism $?^{7}$ Thomson's lamp was supposed to show that a statement about the last state of such a supertask is not possible: we will not be able to tell whether the lamp is on or off after one minute as the button has been pressed infinitely many times. Hence, if supertasks are possible, the chain of causes seems to be undetermined in certain areas.

\footnotetext{
${ }^{4}$ Thomson, J. F., "Tasks and Supertasks", in: Analysis 15, 1954, pp. 1-13.

${ }^{5}$ Cf., for instance, J. F. Thomson for impossibility in both respects, Peter Clark (in oral communication) for possibility in both respects, and Bertrand Russell as claiming logical possibility but physical ("medical" as he calls it) impossibility.

${ }^{6}$ More recently, however, supertasks have been introduced in the context of decision theory, see, for example, the articles of Andrew Bacon (2010), "A paradox for supertask decision makers", in: Philosophical Studies 153, pp. 307-311; and Barrett, J., \& Arntzenius, F. (1999), "An infinite decision puzzle", in: Theory and Decision 46(1), 101-103.

${ }^{7}$ Cf., e.g., Laraudogoitia (1996), Laraudogoitia (1997), p. 53, Earman \& Norton (1998), p. 124, and Laraudogoitia (1999).
} 
Accordingly, it seems that if we assume the kinds of infinity we are familiar with from the realm of mathematics, to have an isomorphic physical counterpart, it is hard to avoid such indeterminacies.

Supertasks come in a variety of versions and have become increasingly refined and ingenious - Pi-machines, José Benardete's "Paradox of the Gods" and Graham Priest's and Stephen Yablo's variations of it, ${ }^{8}$ or Pérez Laraudogoitia's "Beautiful Supertasks". The basic question has, however, stayed the same. The debate started in the 1950's between Max Black, Richard Taylor, J. Watling, and J. F. Thomson, ${ }^{9}$ was rekindled by Laraudogoitia and others in the 1990's, and has been in the literature since then. But the actual founder, it is claimed, is Zeno with his dichotomy paradox. ${ }^{10}$ So let us now look at his paradox.

\section{Zeno's dichotomy paradox}

Aristotle, our main source for Zeno's paradoxes of motion, ${ }^{11}$ discusses these paradoxes in book VI, chapter 9 of his Physics (239b11-14). He does not spell out the dichotomy paradox there, however, but only points at it. Accordingly, this paradox has to be understood with the help of two other passages dealing with this paradox:

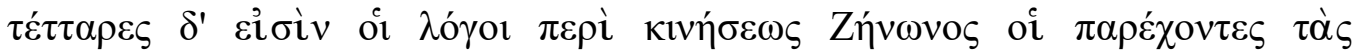

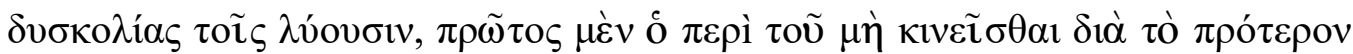

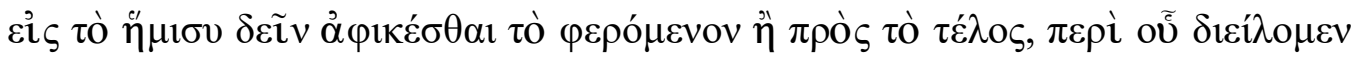

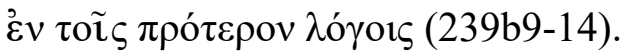

\footnotetext{
${ }^{8}$ Cf. Priest, G. (1999), "On a version of one of Zeno's paradoxes", in: Analysis 59, pp. 1-2; and Yablo, S. (2000), "A reply to new Zeno", Analysis 60(2), pp. 148-151.

${ }^{9}$ Taylor, Richard: (1951) Mr. Black on Temporal Paradoxes. In: Analysis 12, 1951; Watling, J.: (1952) The Sum of an Infinite Series. In: Analysis 13, 1952.

${ }^{10}$ In the supertask debate, the "dichotomy paradox" is meant to refer to what is sometimes also called the runner paradox, the paradox described below. For ancient philosophers, the name "dichotomy paradox" is also sometimes used for one of the plurality paradoxes. And the runner paradox is sometimes called "the stadium", following Aristotle's name for it in the Topics, $160 \mathrm{~b} 7$ (a name which is, however, also used to refer to the fourth of Zeno's paradoxes about masses moving in a stadium). I will stick to the name "dichotomy" in accordance with its usage in the supertask debate and in order to avoid confusion, even if it might not be historically correct (cf. Gregory: Vlastos "Zeno's Race Course", in: Studies in Presocratic Philosophy Vol. II. The Eleatics and Pluralists, ed. R. E. Allen und David J. Furley, London 1975, p. 215, n.2). I will concentrate here on some philosophical points of this paradox important for the debate in question; I give a much fuller discussion of the philological and philosophical problems this paradox raises in my book manuscript The Concept of Motion in Ancient Greek Thought, chapter 3 .

${ }^{11}$ We find paraphrases of the paradox in Simplicius, Philoponus, Themistius and De Lin. Insec. (Lee, Zeno's fragments (1967), fragments 20-22 and 24-25). But as they are very close to Aristotle's report, I will not quote them here.
} 
There are four arguments of Zeno about movement which cause problems for those who want to solve them: first, the one about the "not-moving," on the grounds that before the moved thing gets to the end it must have arrived at half of it; this we have analysed in the preceding investigations [namely in 233a21ff.].

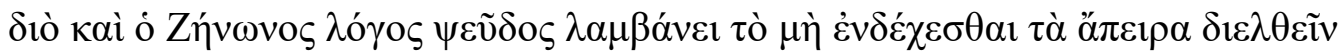

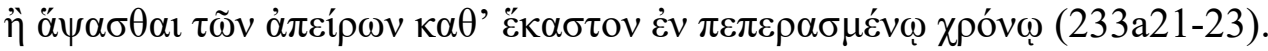

For Zeno's argument turns out to be wrong (in assuming) that it is not possible to go through the infinite or to touch each single (part) of the infinite in a finite time.

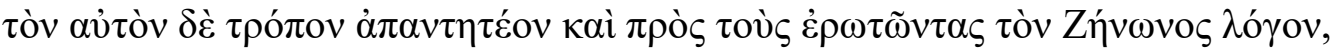

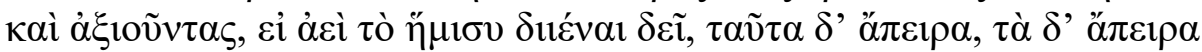

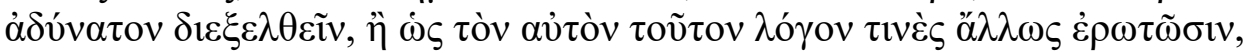

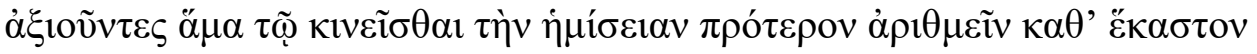

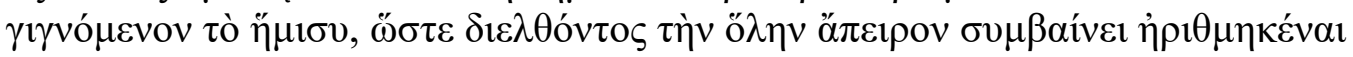

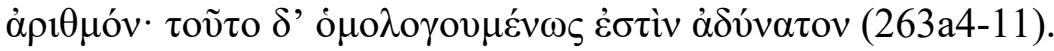

In the same way one has to reply to those who put forth Zeno's puzzle and claim that, if it is always necessary to go through the half (before one can traverse the whole distance), ${ }^{12}$ and these (halves) are infinite (in number), then it is impossible to go through the infinite. Or, as the same argument is asked by some in a different way, at the same time as the movement starts across the half, one could count each single half which occurred before, so that we get the result that if something has travelled the whole (half), we have already counted an infinite number; but this is admittedly impossible.

Taking all three passages together, we can reconstruct the problem raised by Zeno as follows: if something moves over a certain finite distance in a finite time, for example, a runner covering a finite track, he first has to cover half of this distance. Of the second half of the given distance, the runner again has to cover the first half of the remaining distance, then again the first half of the still remaining distance, and so on ad infinitum. So he will have to pass an infinite number of spatial segments before reaching the end. ${ }^{13}$

\footnotetext{
12 Something like these words in brackets has to be understood as the background to make the argument understandable, and is added in all translations. Aristotle does not talk of distance here, only of "something infinite". But when he refers back to this passage in 263a18, he asks how the question posed there would change if length $(\mu \tilde{\eta} \kappa о \varsigma)$ were not taken into account, which justifies the talk about distance or length in the passage quoted.

${ }^{13}$ In 263a4-11 this paradox is presented in two forms, which, in accordance with the usage of the secondary literature, can be called "progressive" and "regressive" (cf.,e.g., Salmon (1980), pp. 32-33, and
} 
It has frequently been claimed that Zeno's paradox thus shows a runner running a finite distance to be performing infinitely many tasks - a supertask. ${ }^{14}$ At least this is how well-known scholars of ancient philosophy, like Jonathan Barnes and Kirk, Raven, and Schofield, have understood it. Barnes for example, sums up the dichotomy paradox as follows: ${ }^{15}$

Zeno thinks to establish: (1) If anything moves, it performs infinitely many tasks. Since he holds it to be a truism that: (2) Nothing can perform infinitely many tasks, he concludes that nothing moves. Unless we are to follow Zeno into his immobile world, we must reject either (1) or (2). Philosophical controversy has settled about (2). ${ }^{16}$

Thus, Barnes understands the paradox exactly as positing the problem of performing infinitely many tasks in a finite time, and thus as understanding motion as a supertask. $\mathrm{He}$ is right that in the past the debate has indeed centred on the possibility of supertasks (2), rather than on the question whether the paradox is indeed presenting motion as a supertask (1). This second point is also the focus of Barnes's discussion. However, I want to demonstrate in the following that the debate concerning Zeno's paradox should settle on (1), on whether the paradox does indeed aim to establish motion as a supertask. More precisely, I want to show, first, that Zeno's paradox does indeed not pose the problem of understanding a run as performing infinitely many tasks, and that, second, we should not try to conceptualize a continuous run as a supertask either.

This will be done by comparing the features of motion presented to us in $\mathrm{Ze}$ no's paradox with the features of a supertask. In order to decide whether the dichotomy paradox can be understood as a supertask, we have to get clearer first on what the decisive features of a supertask are. So let us begin by analysing the notion of a supertask, before we compare supertasks to the way motion is captured in Zeno's paradox.

\footnotetext{
Vlastos (1975a), p. 201). The first variant, the progressive form, is just the given state of affairs. The second, regressive version, shows that the runner must have already gone through an infinite number of spatial segments to cover even the first half of the distance. So while in the first variation a runner can never reach the end of his track, in the second he cannot even get started. As the supertaks debate has focused on the progressive form, this is the one I will also concentrate on here.

${ }^{14}$ There are other problems this paradox raises which I discuss in my book manuscript The Concept of Motion in Ancient Greek Thought, chapter 3.

${ }^{15}$ Barnes (1982), p. 263; he obviously follows Thomson's (1954) summary (without, however, making this clear).

${ }^{16}$ Kirk, Raven, and Schofield's (1983) final comments on the dichotomy paradox sound like a summary of the supertask debate, p. 272: "[...] philosophers cannot agree whether the impossibility of completing the performance of an infinite number of discrete physical acts (if indeed that is impossible) is a logical or merely a physical impossibility, nor what in either case the impossibility consists in."
} 


\section{A Comparison}

\subsection{Analysis of the notion of a task and of a supertask}

The term "supertask" was first introduced into the philosophical conversation ${ }^{17}$ by Thomson in his 1954 paper as referring to an infinity of tasks ${ }^{18}$ and was taken up in the discussion as the notion of an an infinite sequence of actions carried out in a finite time. The notion of a task is, in accordance with it, understood as an action or a finite sequence of actions carried out in a finite time. ${ }^{19}$ However, the definition of an "action" or "act" involved in such a task or supertask did not receive much attention in the debate. Black (1951) understands an act as "something marked off from its surrounding by having a definite beginning and end" and further specifies it in his 1954 paper $^{20}$ in the following way:

Suppose the condition of a body throughout a period of time $\left(t_{1}, t_{2}\right)$ is partially represented by means of a variable magnitude, $m$, which is such that (a) $m$ changes during $\left(t_{1}, t_{2}\right)$, i.e., does not preserve the same value throughout the period, (b), $m$ has "turning points" at both $\mathrm{t}_{1}$ and $\mathrm{t}_{2}$, i.e., has either a maximum or a minimum at $t_{2}$. When these conditions are fulfilled, I propose to say the body in question undergoes a well-bounded change. Now a well-bounded change, when caused by the body itself, is just what was called an "act" in the preceding essay [i.e. the 1951 one].

As an example Black suggests imagining a ball bouncing up and down and replacing the ball then by a man jumping up and down - such a man then can be said to perform a series of acts. ${ }^{21}$ We see that for Black, an act has a clear beginning and end, which are marked by "turning points". Allis and Koetsier (1995) take it that an "action, when applied in a given world state, results in a change of state." Laraudogoitia takes up this account of an action and elaborates it as follows in his SEP article on supertasks:

\footnotetext{
${ }^{17}$ Cf. for example Laraudogoitia (2001), p.1, and Thomson (1954), p.2.

${ }^{18}$ Thomsen did not, however, think we have the concept of a supertask, since for him the idea of completing infinitely many tasks (in a finite time) is inconsistent. And Paul Benecerraf understands the notion of a supertask as a conceptual mismatch.

${ }^{19}$ Cf. Allis and Koetsier (1995) who understand the difference between a task and a super-task informally as follows: "a task consists of a finite sequence of actions, while a supertaks consists of an infinite sequence of actions".

20 "Is Achilles still running", in Problems of Analysis, 1954, p.114.

${ }^{21}$ In this 1954 paper, Black also somewhat changes his position - he thinks that if the height of such jumping converges to zero, so that we get a convergent series of acts, it is no longer clear that it is impossible to perform such an infinite series in a finite time (as this motion does not involve abrogation of continuity at the end point; p. 115). Instead Black now retreats to the weaker position that it is still illegitimate to talk about an infinite series of acts performed in a finite time "due to the 'grammar' of a word like "jump"" (p. 116), claiming that a jump cannot get indefinitely small or brief without ceasing to be a jump. I will mainly deal with his stronger position in the 1951 paper in the main text.
} 
We will assume that at each instant of time the state of the world relevant to a specific action can be described by a set $S$ of sentences. Now an action or operation applied to a state of the world results in a change in that state, that is, in the set $S$ corresponding to it. Consequently, an arbitrary action $a$ will be defined (Allis and Koetsier 1995) as a change in the state of the world by which the latter changes from state $S$ before the change to state $a(S)$ after it. This means that an action has a beginning and an end, but does not entail that there is a finite lapse of time between them.

For Laraudogoitia, an action has a clear beginning and end, but need not require a laps of time between the beginning and the end of a change. He explicitly endorses this assumption by claiming that in principle the individual actions need not be temporally extended, rather they could also be instantaneous actions. This is an account of action that points towards a notion of change in which a change is nothing but a series of different states - a notion which is not only fundamental for the supertask debate, but also at the core of the at-at-theory of motion. ${ }^{22}$ Laraudogoitia also adds that an action need not involve human agency, and indeed the majority of the supertask literature by now has either dealt with machines or particles.

Let us look at a paradigmatic supertask to illustrate the central features of an act or action. In principle, a task can consist of several actions, but it need not; so let us here, for clarity's sake, deal with the case where a task consists of one action only, as is the case with Black's marble transferring machines Beta and Gamma. Here one action is Beta's transfer of a marble from one side A to the other side B. It starts with the beginning of the transfer movement from $\mathrm{A}$ and ends with the coming to a standstill at $\mathrm{B}$, and is thus clearly distinguished from the next action, which is Gamma's transfer of the marble from B to A. Beta's and Gamma's first task each takes a minute, the second one each half a minute, etc. We see here that an action is a single, clearly defined operation that has a definite beginning and end (as claimed by Black and Laraudogoitia). It is strictly distinguished from the next action - either by a change of the direction of movement, or by a standstill in between two tasks, or by an alternating state (what Black (1954) called maximums and minimums). ${ }^{23}$

\footnotetext{
${ }^{22}$ The at-at-theory of motion assumes a series of states, while supertasks are supposed to be a series of actions, but we will see that this does still allow for them displaying an essentially similar structure.

${ }^{23}$ In Black's construction, the movement from left to right is performed by infinity machine Beta while the movement from right to left by the infinity machine Gamma, so different tasks are additionally differentiated from each other by different agents. In the case of Black's infinity machine Alpha, where one infinity machine transfers infinitely many different marbles from one side to the other, different tasks are additionally differentiated from each other by different marbles moved.
} 
A supertask, accordingly, is a series of discrete actions, each of which has a beginning and end, ${ }^{24}$ often an alternating series. No action can be arbitrarily chosen; rather each action is clearly determined by the preceding one. By contrast, the beginning of the whole supertask could be changed without changing anything substantial about the supertask: we could have Black's marble transferring machine start one task earlier, with Gamma's transfer of the marble from B to A, and we would still have a supertask. Equally, Thomson's lamp could start two actions earlier (on after 2 minutes, off after 1 minute) or later (on after $1 / 8$ of a minute, off after $1 / 16$ off a minute). And the end of a supertask is only determined by a set time (after 4 minutes for Black's transferring machine, after 1 minute for Thomson's lamp), but not by any task. It is inherent to a supertask that it cannot have an end defined by a task - after all, supertasks are meant to contain infinitely many tasks, so there is no way that we can define the last task; we can only give a certain point in time as the end point.

Let us now see whether Zeno's paradox does indeed present us with a supertask and whether a continuous movement as discussed in the dichotomy paradox can be understood as such a supertask.

\subsection{Motion as presented in Zeno's paradox}

Zeno's paradox, as it is handed down to us by Aristotle, claims that "before the thing moved gets to the end of its course, it must have arrived at half of it", and before that at half of this half, ad infinitum. So the parts we are dealing with in this paradox are gained from a preceding whole (that is halved, and then halved again, etc. - a process which essentially depends on there being a whole first that can be halved). The whole is given and prior to the parts, since the parts are merely the result of dividing the whole. These parts can be freely chosen, and they are always further divisible (the half is halved again, etc.), which is one of the main problems the dichotomy paradox presents. ${ }^{25}$

\footnotetext{
${ }^{24}$ It can but need not be temporally extended.

${ }^{25}$ The result of this paradox can be spelt out with the help of Zeno's plurality paradoxes (Lee fragment 2 and 3) which show that any way we try to understand the parts resulting from a division of such a whole that is divisible without any restriction leads into difficulties. For they are either $(\alpha)$ parts with nil extension, which raises the question how even an infinite plurality of unextended parts can ever lead to something physically extended; or $(\beta)$ the parts have some extension, in which case it seem unclear in a Zenonian context why an infinite number of these physically extended parts does not lead to an infinitely extended whole; or $(\gamma)$ we do not commit ourselves to either possibility, but claim that we could just go on with the division ad infinitum, in which case we are facing the problem that the parts are left indeterminate. We can see how all three possibilities may seem problematic, even if all three
} 
Accordingly, the dichotomy paradox confronts us with the problem of how to think about the possible parts of a continuous motion, which are not simply given but can be freely chosen and are always divisible into further parts. Whereas with a supertask the parts - the individual tasks - are given and clearly defined, with the finite run, as presented in the dichotomy paradox, beginning and end are determined, but the parts are not. Accordingly, the way movement is presented in the dichotomy paradox is not as a series in the way a supertask is, since it does not have any given, discrete parts. $^{26}$ There is nothing like rest, a change of direction, or anything similar that would clearly separate one part of the run from the next one. Rather we can mark off different parts of the run as we please and always divide them further.

There have, however, been several attempts in the secondary literature to model Zeno's dichotomy explicitly after a supertask by introducing ways of transforming parts of the run into given parts that are thus clearly separate from each other. Most prominently, Adolf Grünbaum introduced a so-called staccato runner who pauses after the completion of each part of a run. ${ }^{27}$ The runner runs half the distance, then sits down, and relaxes a bit, before he covers a quarter of the distance, a little pause again, an eighth of a distance, etc. In this way the run of the dichotomy paradox seems to be a supertask after all. For it seems all Grünbaum does by introducing these pauses is to bring out the fact that the run has parts - he is making this just more obvious by clearly separating each part from the next with the help of a pause. However, by breaking up one movement into such discrete parts, Grünbaum in fact changes the situation in question fundamentally. Instead of dealing with one uninterrupted and thus continuous motion and its possible parts, as in Zeno's paradox, we are now dealing with an infinity of different runs. Grünbaum does not give any reason why this myriad of single runs, interrupted by pauses, should still be considered as one run, rather than as a series of different and independent runs. It seems that each individual

\footnotetext{
have at times been embraced by the tradition following Zeno. This trilemma is not meant as an analysis of the runner paradox itself, but rather shows the problematic consequences for the notion of parts it seems to leave us with.

${ }^{26}$ Barnes (1982) p. 264 admits that the notion of a task does not appear in our account of Zeno's paradox: "for it is mildly odd to call each of $b$ 's successive moves to the next $a_{\mathrm{i}}$ a 'run' or a 'task'. Yet the point is merely verbal: Zeno himself does not use the terminology". But as Barnes believes that the runner has infinitely many subsections of $\mathrm{AB}$ to traverse, he thinks the "claim that $b$ 's operations are not 'tasks', or are only 'tasks' in a Pickwickian sense" to be "boring". The problem is, however, not boredom, but that we are changing the conception of the very structure of continuous motion as presented to us in the paradox if we understand it as a sequence of runs.

${ }^{27}$ Grünbaum (1968), p.79. The staccato runner is taken up again by Salmon (1980), p. 47; Barnes (1982), p. 267; and Moore (1990), pp.3-4.
} 
little run can be considered as a continuous motion, but that the series of runs he thus constructed very clearly cannot be constructed to be continuous.

And we are getting very different kinds of parts: in Grünbaum's scenario we are dealing with parts whose identity is clearly fixed by two surrounding stretches of rest, while in Zeno's case we are dealing with parts whose identity rests only on our mind marking them off and which can easily be changed (for example, we would get into the very same paradox if we claimed to mark off a third of the given whole, and a third of the third, etc.). Thus, Grünbaum and Zeno are actually talking about two different problems - Zeno talks about a continuous run, Grünbaum about a series of discrete states. ${ }^{28}$

We may be tempted to think that whatever is the case with the dichotomy, Zeno's paradox of Achilles and the tortoise, nevertheless, seems to be a supertask: this latter paradox attempts to show that when Achilles starts behind the slowest one in a race, a tortoise as later authors have it, it seems impossible that he will ever overtake the tortoise. For first, Achilles has to cover the distance from his starting point, A, to the starting point of the tortoise, B. In the meantime the tortoise has moved on to another point $\mathrm{B}_{1}$, and when Achilles reaches this next point $\mathrm{B}_{1}$ the tortoise will have moved ahead again to $B_{2}$. But while the distances between the successive Bs will increasingly diminish, it seems that no matter which $\mathrm{B}_{\mathrm{n}}$ Achilles has reached, the tortoise will always have moved to $B_{n+1}$. It seems that here there is no given whole from which the parts are derived; and Achilles has to perform an infinite series of tasks in order to level with the tortoise.

However, as Aristotle has already pointed out in his Physics, 239b18ff., from a logical point of view, this paradox is simply a variation of the dichotomy paradox. ${ }^{29}$ The whole that gets divided in this paradox is the distance between Achilles' starting point $\mathrm{A}$ and the possible point $\mathrm{C}$ where Achilles and the tortoise are at the same level (even if this last point does not belong to the points reached by the construction

\footnotetext{
${ }^{28}$ Black, Max, "Achilles and the Tortoise", in: Analysis 11 (1951) and Thomson (1954) draw the conclusion that the assumption of completing infinitely many tasks leads to absurdities, and thus is logically not possible. Accordingly, they reason that the run in Zeno's paradox cannot be correctly described as completing an infinite sequence of tasks as it is obviously physically possible and so needs another conceptualisation. Salmon (1980), on the other hand, thinks that some small modifications, as, e.g., letting the distance which the button of Thomson's lamp is moved during each switch converge to zero, are enough to make the infinity machine logically possible and of the same logical structure as the run of a finite distance, see pp. 46-48.

${ }^{29}$ Many modern scholars follow this assessment; cf. Barnes (1982), p. 274, Kirk, Raven, and Schofield (1983), p. 272, and Ferber (1995), pp. 8-10.
} 
sketched in the paradox). While in the runner paradox the distance was divided by halving it, here the track is divided by the tortoise's motion, which Achilles then "inherits". Again, we do not have discrete tasks that the tortoise performs and that are distinguished from each other by periods of rest, change of direction, or something of this kind. Rather, we have a continuous motion of the tortoise, and at certain times (when Achilles has reached the point the tortoise left) we look how far it has already proceeded.

We should also note that the basic paradox is independent of whether the division is regular or not, for the tortoise could move in an irregular way (slower at some times, a bit faster at others within a certain range) and we would still get the same paradox. By contrast, supertasks depend on a regular division, since only such a division can be said to converge to a certain limit, while Zeno's paradox would also works with irregular divisions.

\section{3 The unity of a continuous motion}

Zeno's dichotomy paradox invites us to think about what exactly the relation is between the whole of a run and the parts that we can gain from it. But it does not treat a continuous run as a supertask, which is a clear advantage if we want to understand continuous processes. For in order to conceptualize continuous processes, and that means processes that are necessarily temporally extended, thinking of them in terms of supertasks leads us down a mistaken path.

Now Zeno presents us with a paradox, the paradox that in order to cover a finite distance in a finite time it seems we need to cover infinitely many spatial parts. Given that it is a paradox, we'd better be cautious not to take his account of motion at face value, for we know that in so far as it is a paradox something has to be wrong with this conceptualization of motion - there is another mistaken path here. And when we try to conceptualize continuous motion, we do not have to use division into two as the principle of division, as is the case in the set up of the dichotomy. In principle, parts can be freely chosen as we please. However, the way motion is presented in the dichotomy paradox gives us an understanding of the part-whole structure of a continuous motion that is an important alternative to the one we encounter with supertasks, and one that is worth exploring further.

Thinking of continuous processes as supertasks seems to be supported by having come to think of processes as nothing but a series of states of being at a particular 
place (or in a particular condition) at a particular time - along the lines of the socalled at-at-theory of motion prevalent since Russell in metaphysical debates (who prominently used it in his discussion of Zeno's arrow paradox). ${ }^{30}$ Such an understanding of processes prepares the ground for introducing the notion of a supertask as it conceptualizes processes as a series consisting of given discrete elements. If we combine this understanding with the mathematical notion of a convergent geometrical series, i.e. with the mathematical idea that the sum of infinitely many summands that get smaller and smaller is finite, we arrive at the idea that continuous processes may be captured as supertasks (if supertasks are indeed possible).

However, looking at the differences I have pointed out between Zeno's dichotomy paradox and supertasks, we may start to doubt whether processes like a continuous run can indeed be genuinely conceptualized as supertasks. For continuous processes do not seem to share their main structure with supertasks. A supertask is a series in which each part is defined while the beginning and the end of the whole are undetermined. The clear division between two parts (tasks) is a necessary characteristic of the series "supertask" - the division is due to the change of direction, or the change from a maximum to a minimum, etc., so there is no other division possible. But such a division is not a characteristic of the "series" "run". However, if not even a single action making up a task is clearly marked within a continuous motion, it seems hard to define the whole movement as a supertask. Continuous motion seems to have essentially a different mereological structure, a different part-whole relation, than a supertask has, and thus to raise different questions about its unity: supertasks seem to work with a weak notion of a whole, for, as we saw earlier, nothing changes for the supertask if we start a few tasks earlier or later. By contrast, continuous motions seem to involve a weak notion of a part, since they can be freely chosen. ${ }^{31}$ Not all parts we can choose are disjoint. For example, if I chose as parts the first three quarters of the whole as well as the last two thirds, these parts will overlap, and thus not be disjoint. We can, however, chose disjoint parts, as, for example, the first quarter and the last third of the whole. But even if we chose distinct and disjoint parts of a run, the identity of these parts is not given, as it is in the supertask case, and the whole run does not settle their identity. Accordingly, while we can choose distinct parts, they will not be

\footnotetext{
${ }^{30}$ Cf. also Salmon (1975), Space, Time, and Motion, pp. 40-42.

31 This understanding of continuous motion fits with Aristotle's, which I discuss in detail in my book manuscript The Concept of Motion in Ancient Greek Thought, chapter 7.
} 
separate parts in the sense that a clear mechanism (like a change of direction or a standstill) marks them off from each other and fixes their identity. If somebody were to ask "but isn't the runner running each of the parts of the whole", we would have to answer: what do you understand by "each of its parts"? The overlapping as well as the non-overlapping ones? Each possible part somebody might choose? Such a question, it seems, presupposes that the parts are somehow already given, and thus can or cannot be covered.

Hence, I agree with Black and Thomson that continuous motion as described in the dichotomy paradox cannot be understood as a supertask, though for a different reason: not, as Black and Thomson suppose, because supertasks are logically impossible (since the last state of the series cannot be determined) - in the present paper I remain neutral on this question. But rather because the actions of a supertask have to be understood quite differently to the parts of a run: with a finite run beginning and end are clearly defined, while parts can be chosen as we please, whereas with a supertask beginning and end can vary, while each task (each part) is clearly defined.

But if we cannot think of continuous processes as supertasks, we should also reconsider what has supported this assumption in the first place, namely the so-called at-at-theory of motion. This theory is often helpful to describe a motion or change something changes from being $F$ at $t_{1}$ to not-being $F$ at $t_{2}$ - especially if we want to perform mathematical calculations. But this way of describing motion leaves out an important feature, the very feature of physical continuity. And if we want to conceptualize continuous motion in a way that also captures the difference to a series of discrete snapshots, we'd better not understand motion as consisting ${ }^{32}$ in being at a particular point at a particular time. ${ }^{33}$

Note that if I point out the difference in the merelogical structure between the description offered by the at-at-theory and continuous motion, I am not thus claiming that all motion has to be continuous - there may very well be series of discrete jumps,

\footnotetext{
${ }^{32}$ Black (1954), p. 125, n.18 also points out that even if a mathematical description is correct, this does not yet demonstrate that "each distinguishable item of the mathematical description must be in a oneto-one correspondence with distinguishable items of physical reality".

${ }^{33}$ As, for example, Salmon does, who in his account of Zeno's arrow paradox claims that "[...] motion itself is described by the pairing of positions with times alone. [...] the motion consists in being at a particular point at a particular time" (p. 41, his italics). We see that he switches, without any explanation or justification, from talking about how "motion itself is described" to "motion consists in being at a particular point at a particular time". From the fact that in mathematics motion can be described as the "pairing of positions with times" he infers "motion to be composed of immobilities," ascribing the mathematical concept to motion itself.
} 
for example at the subatomic level; but this is not the motion I am concerned with here.

\section{Conclusion}

Summing up, we can say that the dichotomy paradox and supertasks raise genuinely different questions about processes. Looking at these differences, we may think that this is not just a historic accident, but that there are indeed essential differences between a finite continuous run and supertasks in the kind of unity they require. In the case of a supertask, we start out with given discrete states, the individual actions, which constitute the whole of a supertask. The unity of such a supertask is based on the rule that the same task has to be performed in a constantly diminishing time, while beginning and end are determined externally by the time fixed. By contrast, the unity of a continuous motion over a certain finite distance is not defined by any rule determining a series of fixedly given tasks, but rather by the phases of rest which mark the beginning and end of the movement. In this case, neither are there any parts given nor are these parts fixed once some parts have been freely chosen, since there is no better reason to determine a chosen piece as a part than to take, for instance, the halves of this piece as parts.

So while with a continuous finite run, beginning and end are determined but the parts can be chosen as one pleases, arbitrarily, with a supertask it is the other way around: each single task, and thus each part, is determined, while the beginning and the end of the whole supertask can be chosen. And with supertasks there can be pauses between each task, since the absence of rest is not important for the unity of a supertask, whereas it is essential for the unity of a continuous motion.

Zeno's paradox forces us to think about what kind of whole a continuous run is. And comparing supertasks with Zeno's paradoxes helps to see that continuous motion presents us with a genuinely different part-whole relation than supertasks do this is the philosophically central point of the comparison, since a correct understanding of the mereological structure of motion is crucial for its adequate conception. The at-at-theory of motion is one reason why this difference tends to be overlooked.

Accordingly, while Zeno might have inspired the supertask debate and can be seen as its precursor in this sense, he did not start the debate. For the central question he puts forward - how can we think about a continuous motion where parts can be derived from the whole that are always further divisible and any attempt to conceptu- 
alize such parts seems to be problematic - is different from the one the supertask debate raises.

So whether or not we think supertasks are possible, whether or not we think that supertasks are confused concepts, if we understand a run as an infinite series of tasks we are not understanding it as a continuous motion any longer. Now there might be contexts where we want to use the notion of a supertask for describing ${ }^{34}$ a motion nevertheless; but we should be aware that characterising something continuous like a run as a supertask means missing out on the specific unity required for conceptualising continuous motion. And we should thus also reconsider our usage of the at-attheory of motion - while it is helpful for calculations, it may not be an adequate conceptualization of motion.

Whereas Thomson and Black were reluctant to understand the dichotomy as a supertask, the later supertask debate got rid of any doubts and took this paradox as the founding father of all supertasks. The reason, I think, the dichotomy is understood as a supertask, lies in a lack of analysis of what is actually understood by the actions that are seen as making up a supertask. The mathematical notion of a convergent geometrical series, where the sum of infinitely many summands is finite, seems to have been applied to a variety of physical phenomena without first clarifying whether this mathematical notion can indeed be applied in all these cases. So the first question with respect to a continuous run should not be how such a supertaks is logically and physically possible, but rather whether the notion of a supertask can indeed be applied to continuous motion, and what is specific for continuous motion that has to be taken into account so as not to apply something inappropriately. ${ }^{35}$

\footnotetext{
${ }^{34} \mathrm{Cf}$. also Black for this difference and my book manuscript The Concept of Motion in Ancient Greek Thought, chapter 3 .

${ }^{35}$ I want to thank Peter Clark for first introducing me to the supertaks debate. The initial version of the paper also profited a lot from discussions with Uli Bergman.
} 